\title{
Darf man Patientenrechte mit Limitationen im Tarif beschneiden?
}

\section{Dr. med. Urs Stoffel}

Mitglied des Zentralvorstandes, Departementsverantwortlicher Ambulante Versorgung und Tarife

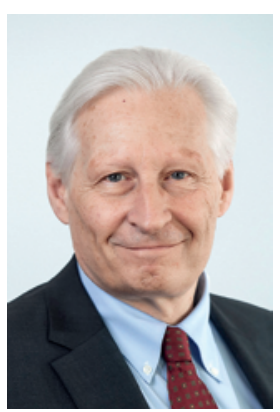

Um die Antwort gleich vorwegzunehmen: Die Tarifpartner dürfen in einem Tarif keine Limitationen festlegen, welche einen an sich bestehenden gesetzlichen Leistungsanspruch des Patienten beschränken. Und schon gar nicht solche, die einzig und allein dazu dienen, Mengenausweitungen zu verhindern. Dafür gibt es seit der Einführung des TARMED andere gesetzliche Grundlagen und Mechanismen.

Im Krankenversicherungsgesetz (KVG), welches die Grundlage des ambulanten Tarifs TARMED ist, geht man von der Pflichtleistungsvermutung aus, d.h., dass die ärztliche Krankheitsbehandlung grundsätzlich den Prinzipien der Wirksamkeit, Wirtschaftlichkeit und Zweckmässigkeit (WZW) entspricht, solange der Bundesrat sie nicht von der Kostenvergütungspflicht ausnimmt oder an bestimmte Bedingungen knüpft. Der Patient hat somit grundsätzlich Anspruch auf diese Pflichtleistungen.

Der Anspruch des Patienten auf eine Pflichtleistung kann nicht über eine Limitation der Leistung im Tarif beschränkt werden.

In zwei Rechtsgutachten, welche zur Frage des «Globalbudgets und der Tarifgestaltung in der Krankenversicherung $»^{1}$ und zur Frage der "echten Mehrleistungen im ambulanten Bereich ${ }^{2}$ juristisch Stellung nehmen, wird die Aussage bestätigt, dass man Pflichtleistungen nach dem KVG nicht über Limitationen im Tarif beschränken kann.

Der Anspruch des Patienten auf eine Pflichtleistung kann daher nicht über eine Limitation der Leistung im Tarif beschränkt werden. Eine solche Begrenzung könnte an sich ausschliesslich der Bundesrat festlegen. Diese Limitationen dürften dann aber nicht im Tarif festgelegt, sondern müssten in der KrankenpflegeLeistungsverordnung (KLV) verankert werden, und vor allem müssten sie auch die WZW-Kriterien erfüllen. Sämtliche Leistungen, welche in der KLV definiert sind, müssen auch die WZW-Kriterien gemäss Art. 32 KVG erfüllen.

Das Bundesamt für Justiz (BJ) geht sogar noch weiter und hat gegenüber dem Bundesamt für Gesundheit BAG festgehalten, dass es nicht vorgesehen ist, dass Leistungserbringer und Kostenträger in der Tarifstruktur gemeinsam Limitationen von Pflichtleistungen festlegen.

\section{Eine Limitation von Zeitleistungen im}

ambulanten Tarif, welche einzig und allein der Verhinderung von Mengenausweitung dient,lehnt die FMH kategorisch ab.

Damit verbietet das BJ geradezu den Tarifpartnern, in der Tarifstruktur Pflichtleistungen zu limitieren. Solche Limitationen können vom Bundesrat nur über die KLV - nicht aber über den Tarif - eingeführt werden. Die FMH hat sich vor allem auch in den Tarifverhandlungen immer klar geäussert: Die Limitation von Zeitleistungen im ambulanten Tarif, welche einzig und allein der Verhinderung von Mengenausweitung dient und damit rein ökonomisch begründet ist, wird kategorisch abgelehnt.

Im Hinblick auf das Thema der Mengenausweitung hat sich eines der bereits erwähnten Gutachten ebenfalls klar geäussert: Bei ungerechtfertigten Mehrleistungen im Sinne einer Mengenausweitung sind gesetzliche Mechanismen vorgesehen, welche gemäss Art. 56 KVG ausschliesslich über die Anwendung der WZWKriterien Missbrauch verhindern können oder sogar Rückforderungen zulassen.

Die FMH unterstützt deshalb Limitationen nur, wenn diese medizinisch indiziert sind, aufgrund von gesetzlichen Vorgaben (z.B. Strahlenschutz) erlassen werden oder dem Schutz der Patientinnen und Patienten dienen. Solche Limitationen sind sachgerecht und werden auch von den angeschlossenen Ärzteorganisationen unterstützt und befürwortet. 